\title{
ON THE ABSENCE OF COHOMOLOGICAL FINITENESS IN WREATH PRODUCTS
}

\author{
GILBERT BAUMSLAG, MARTIN R. BRIDSON and KARL W. GRUENBERG
}

(Received 14 July 1997)

Communicated by J. R. J. Groves

\begin{abstract}
The wreath product $W=A \geq T$, where $A \neq 1$, is of type $F P_{2}$ if and only if $T$ is finite and $A$ is of type $F P_{2}$.

1991 Mathematics subject classification (Amer. Math. Soc.): primary 20E22, 20F05, $20 \mathrm{~J} 05$.
\end{abstract}

\section{Introduction}

In 1980, Bieri and Strebel proved that every finitely generated metabelian group of type $F P_{2}$ is finitely presented [5]. This shows that, in this restricted setting, the most natural cohomological and geometric finiteness conditions are equivalent in dimension two. The question as to whether these conditions are equivalent in general remained unanswered until recently, when Bestvina and Brady constructed a class of counterexamples by using Morse theory for cubical complexes [3]. They also obtained a number of positive results, couched in geometric terms.

The purpose of the present note is to point out that one cannot build counterexamples by means of familiar algebraic constructions such as wreath products, HNN extensions, free products with amalgamation and the like. For graphs of groups this follows from a standard argument using an appropriate Mayer-Vietoris sequence (see [4]) - this fact was pointed out to us by Peter Kropholler. In particular, an amalgamated product of two finitely presentable groups is of type $F P_{2}$ if and only if it is finitely presentable (see [1]). Here we will prove the following theorem.

The second author is an EPSRC Advanced Fellow. This work was also supported in part by NSF Grants \#9103098 (first author) and \#9401362 (second author).

(C) 1998 Australian Mathematical Society 0263-6115/98 $\$ A 2.00+0.00$ 
THEOREM 1. The wreath product $W=A \geq T$, where $A \neq 1$, is of type $F P_{2}$ if and only if $T$ is finite and $A$ is of type $F P_{2}$.

The argument that we will give here is modelled in part on the proof of an analogous result [2] where $F P_{2}$ is replaced by finitely presentable.

\section{Preliminaries}

2.1. Some notation and definitions We use the notation $[x, y]=x^{-1} y^{-1} x y$ and $a^{t}=t^{-1} a t$ throughout this note; and we write $[G, G]$ for the commutator subgroup of a group $G$.

Let $U$ be a fixed group. Then a group $V$ is termed a $U$-group if $V$ comes equipped with a homomorphism of $U$ into the automorphism group of $V$. The class of $U$ groups constitutes a category in the usual way. If $S$ is a subset of $V$, then we denote the intersection of all $U$-subgroups of $V$ containing $S$ by gp $U$ (S). If $W$ is a $U$-subgroup of $V$, then we denote the minimal cardinality of those sets $S$ such that $\operatorname{gp}_{U}(S)=W$ by $d_{U}(W)$, provided it is finite; otherwise we define $d_{U}(W)=\infty$. If $U=1$, we simply denote $d_{U}(W)$ by $d(W)$-in this case $d(W)$ is the minimal number of generators of the subgroup $W$ of $V$. Observe that if the $U$-group $\bar{V}$ is a quotient of the $U$-group $V$, then

$$
d_{U}(\bar{V}) \leq d_{U}(V)
$$

Recall that a group $G$ is of type $F P_{n}$ if there is a projective resolution

$$
\cdots \rightarrow P_{n} \longrightarrow P_{n-1} \cdots \longrightarrow P_{1} \longrightarrow P_{0} \longrightarrow Z
$$

of the trivial left $G$-module $Z$ with $P_{n}, \ldots, P_{0}$ finitely generated projective $G$-modules. It turns out that a group $G$ is of type $F P_{1}$ if and only if it is finitely generated. Consequently if a group $G$ is of type $F P_{2}$, then $G$ must be finitely generated. In this case we can represent $G$ as a factor group of a finitely generated free group $F$ :

$$
G \cong F / K \text {. }
$$

Since $K$ is a normal subgroup of $F$, we can view it as an $F$-group, where $F$ acts by conjugation. Then $\bar{K}=K /[K, K]$ is a quotient $F$-group of the $F$-group $K$. It then turns out that $G$ is of type $F P_{2}$ if and only if

$$
d_{F}(\bar{K})<\infty
$$

for all choices of the finitely generated free group $F$ and the various normal subgroups $K$ that are involved. (See Proposition 2.2 on page 20 of [4].)

We shall need to make use of these characterisations of $F P_{1}$ and $F P_{2}$ when we come to the proof of the theorem. 
2.2. Sketch of the proof of the theorem Suppose that

$$
W=A \imath T
$$

where $A \neq 1$ and $T$ is infinite. The interesting part of the theorem is the proof that, under these circumstances, $W$ cannot be of type $F P_{2}$.

The first step is to show that if $W$ is of type $F P_{2}$ then both $A$ and $T$ must be finitely generated. Next, if

$$
A=\langle X ; R\rangle, \quad T=\langle Y ; S\rangle
$$

are presentations of $A$ and $T$ with $X$ and $Y$ finite, we choose $F$ to be the free group on $X \cup Y$. Furthermore, let

$$
Q=\left\{\left[x, z^{w}\right] \mid x, z \in X, \quad w \text { any Y-word different from } 1 \text { in } T\right\} .
$$

Finally, let

$$
K=\mathrm{gp}_{F}(Q \cup R \cup S)
$$

where here $F$ acts on $F$ by conjugation - so $K$ is the normal closure in $F$ of $Q \cup R \cup S$. It follows then that

$$
W \cong F / K
$$

Our objective is to prove that $d_{F}(\bar{K})$ is not finite. In fact we will prove that $d_{F}(K / J)=$ $\infty$, where

$$
J=\mathrm{gp}_{F}([K, K] \cup R \cup S) .
$$

Since $K / J$ is a quotient of $\bar{K}$, this suffices to prove that $W$ is not of type $F P_{2}$. The reason that this approach is manageable stems from the fact that $F / J$ is what Smelkin [9] terms a metabelian wreath product (see Section 4).

\section{Metabelian products}

\subsection{The definition}

DEFINITION 1. Let $\left(A_{\lambda} \mid \lambda \in \Lambda\right)$ be a family of groups and let $B$ be a group. A family of homomorphisms

$$
\left(\psi_{\lambda}: A_{\lambda} \rightarrow B \mid \lambda \in \Lambda\right)
$$


is termed a metabelian family if

$$
1=\left[\left[\psi_{\lambda_{1}}\left(a_{\lambda_{1}}\right), \psi_{\lambda_{2}}\left(a_{\lambda_{2}}\right)\right]^{w},\left[\psi_{\lambda_{3}}\left(a_{\lambda_{3}}\right), \psi_{\lambda_{4}}\left(a_{\lambda_{4}}\right)\right]\right]
$$

for all $\lambda_{1}, \lambda_{2}, \lambda_{3}, \lambda_{4} \in \Lambda$ with $\lambda_{1} \neq \lambda_{2}$ and $\lambda_{3} \neq \lambda_{4}$, all $a_{\lambda_{i}} \in A_{\lambda_{i}}$ and all $w \in$ $\operatorname{gp}\left\{\psi_{\lambda}\left(A_{\lambda}\right) \mid \lambda \in \Lambda\right\}$.

DEFINITION 2. The group $B$ is termed the metabelian product of the family $\left(A_{\lambda} \mid\right.$ $\lambda \in \Lambda)$ if there exists a metabelian family $\left(\psi_{\lambda}: A_{\lambda} \longrightarrow B \mid \lambda \in \Lambda\right.$ ) of homomorphisms into $B$ such that for every group $B^{\prime}$ and every metabelian family $\left(\psi_{\lambda}^{\prime}: A_{\lambda} \longrightarrow B^{\prime} \mid \lambda \in \Lambda\right)$, there exists a unique homomorphism $\Psi: B \longrightarrow B^{\prime}$ such that

$$
\Psi \psi_{\lambda}=\psi_{\lambda}^{\prime} \text { for every } \lambda \in \Lambda
$$

The notion of a metabelian product as well as the more general notion of a verbal product was first introduced by Golovin [6].

It is easy to see, from general principles, that $B$ is uniquely defined by the above universal property. It is also easy to see that each $\psi_{\lambda}$ is monomorphism and, identifying $A_{\lambda}$ with its image in $B$, that $A_{\lambda} \cap A_{\mu}=1, \lambda \neq \mu$. Moreover, $B$ is generated by the subgroups $\left\{A_{\lambda} \mid \lambda \in \Lambda\right\}$. We express the fact that $B$ is the metabelian product of the family $\left(A_{\lambda} \mid \lambda \in \Lambda\right)$ by writing

$$
B=\prod_{\lambda \in \Lambda}^{(2)} A_{\lambda} .
$$

We shall need here also the free product and the restricted direct product of such a family $\left(A_{\lambda} \mid \lambda \in \Lambda\right)$ of groups, which we denote, respectively, by

$$
\prod_{\lambda \in \Lambda}^{(\infty)} A_{\lambda} \text { and } \prod_{\lambda \in \Lambda}^{(1)} A_{\lambda} .
$$

If $L$ is the kernel of the canonical homomorphism of $\prod_{\lambda \in \Lambda}^{(\infty)} A_{\lambda}$ onto $\prod_{\lambda \in \Lambda}^{(1)} A_{\lambda}$, then it is not hard to see that

$$
\prod_{\lambda \in \Lambda}^{(2)} A_{\lambda} \cong\left(\prod_{\lambda \in \Lambda}^{(\infty)} A_{\lambda}\right) /[L, L] .
$$

3.2. An important lemma The following simple lemma is important in the proof of our theorem. 
LEMMA 1. Let

$$
B=\prod_{\lambda \in \Lambda}^{(2)} A_{\lambda}
$$

be the metabelian product of the family of groups $\left(A_{\lambda} \mid \lambda \in \Lambda\right)$. Then the following hold.

(1) If $\Lambda^{\prime}$ is a subset of $\Lambda$, then

$$
\operatorname{gp}\left(A_{\lambda} \mid \lambda \in \Lambda^{\prime}\right)=\prod_{\lambda \in \Lambda^{\prime}}^{(2)} A_{\lambda}
$$

(2) The homomorphism

$$
\text { from } \prod_{\lambda \in \Lambda}^{(2)} A_{\lambda} \text { to } \prod_{\lambda \in \Lambda^{\prime}}^{(2)} A_{\lambda}
$$

which maps $A_{\lambda}$ identically to $A_{\lambda}$ if $\lambda \in \Lambda^{\prime}$ and otherwise to the identity, is a retraction. (3) If $\lambda, \mu \in \Lambda, \lambda \neq \mu$, then for all non-trivial elements $a \in A_{\lambda}, b \in A_{\mu}$,

$$
[a, b] \neq 1
$$

PROOF. The assertion (1) follows immediately from the universal nature of $\prod_{\lambda \in \Lambda}^{(2)} A_{\lambda}$ and (2) is a consequence of this remark. Finally, in the light of either (1) or (2), it suffices to consider the case where $\Lambda=\{1,2\}$. In this case, if $P$ is the free product of $A_{1}$ and $A_{2}$, then $\left[A_{1}, A_{2}\right]$ is the free group, freely generated by the set

$$
\left\{[a, b] \mid a \in A_{1}, b \in A_{2}, a \neq 1, b \neq 1\right\} .
$$

(This is essentially due to Levi [8] - see [7].) So, in view of (2), $[a, b] \neq 1$ in $B$. This completes the proof of the lemma.

\section{Wreath-like products}

Suppose that $G$ is a group and that $A$ and $T$ are subgroups of $G$. We term $G$ a product of $A$ and $T$ if

$$
G=\operatorname{gp}(A \cup T)
$$

We shall have need of three such products, which depend for their definition on the nature of the normal closure $B=\operatorname{gp}_{G}(A)$ of $A$ in $G$. With this in mind, we denote the 
conjugate $t^{-1} A t$ of $A$ by the element $t \in T$ by $A$, and the conjugate $t^{-1}$ at of $a \in A$ by $t \in T$ by $a_{t}$. Then $B$ is generated by the $A_{t}(t \in T)$. If

$$
B=\prod_{t \in T}^{(\infty)} A_{t}
$$

then it is not hard to see that $G$ is simply the free product of $A$ and $T$. In this event we denote $G$ by $G_{\infty}, B$ by $B_{\infty}$ and observe that we have a semidirect product

$$
G_{\infty}=B_{\infty} \cdot T
$$

The remaining two products have similar descriptions. The first of these is the wreath product of $A$ by $T$. In this case

$$
B=\prod_{t \in T}^{(1)} A_{t}
$$

and is denoted by $B_{1}, G$ is denoted by $G_{1}$ and

$$
G_{1}=B_{1} \cdot T=A ; T .
$$

Finally, if

$$
B=\prod_{t \in T}^{(2)} A_{t}
$$

is the metabelian product of the subgroups $A_{t}$ of $G$, then we denote $B$ by $B_{2}, G$ by $G_{2}$ and we observe that

$$
G_{2}=B_{2} \cdot T
$$

which we denote by

$$
A \imath_{2} T \text {. }
$$

Observe that in every one of the cases above $s^{-1} a_{t} s=a_{t s}$. It follows from Lemma 1, that if $a, b \in A, a \neq 1, b \neq 1$ and if $s, t \in T, s \neq t$, then $\left[a_{s}, b_{t}\right] \neq 1$ in $A 2_{2} T$.

\section{The proof of the theorem}

5.1. The easy part of the proof Suppose that $W=A \imath T$, where $A \neq 1$ and $T$ is finite. If $A$ is of type $F P_{2}$, then, adopting the notation introduced above, so too is $B_{1}$. Since $B_{1}$ is of finite index in $W$, it follows that $W$ is also of type $F P_{2}$. 
5.2. Finitely generated wreath products Suppose now that $W$ is of type $F P_{2}$. Then $W$ must be finitely generated. Now if $X$ and $Y$ are generating sets for $A$ and $T$ respectively, then $X \cup Y$ generates $W$. So there are finite subsets $X_{1}$ and $Y_{1}$ of $X$ and $Y$ respectively, such that $X_{1} \cup Y_{1}$ generates $W$. It follows that $X_{1}$ generates $A$ and $Y_{1}$ generates $T$, for example by considering the canonical homomorphims onto $A$ and $T$ via the direct product $A \times T$.

5.3. The case where $A \neq 1$ and $T$ is infinite The main step in the proof of our theorem is the following

LEMMA 2. Let A be a non-trivial, finitely generated group and let $T$ be an infinite, finitely generated group. Then $W=A \geq T$ is not of type $F P_{2}$.

Let us suppose that $W$ is of type $F P_{2}$. Our objective, then, is to derive a contradiction to this assumption.

We adopt the notation introduced in 2.2 and 3.1. Consider then the homomorphism $\theta$ of $F$ onto $G_{\infty}$ which maps $X$ onto the corresponding set of generators of $A$ and $Y$ onto the corresponding set of generators of $T$. Notice that $K$ maps onto $L$ and hence $[K, K]$ maps onto $[L, L]$. It follows that $\theta$ induces a homomorphism of $F /[K, K]$ onto $G_{2}=A l_{2} T$ which maps $\bar{K}$ onto the image, say $L_{2}$, of $L$ in $G_{2}$. As $L_{2}$ is a quotient of $\bar{K}$, it is the normal closure in $G_{2}$ of a finite set. Now

$$
L_{2}=\mathrm{gp}_{G_{2}}\left\{\left[a_{s}, a_{t}^{\prime}\right] \mid a, a^{\prime} \in A, \quad s, t \in T . \quad s \neq t\right\} .
$$

It follows that there is a finite subset $S$ of $T$ such that

$$
L_{2}=\mathrm{gp}_{G_{2}}\left\{\left[a_{s}, a_{t}^{\prime}\right] \mid a, a^{\prime} \in A, \quad s, t \in S, \quad s \neq t\right\} .
$$

It follows therefore also that

$$
L_{2}=\operatorname{gp}_{B_{2}}\left\{\left[a_{s u}, a_{t u}^{\prime}\right] \mid a, a^{\prime} \in A, \quad s, t \in S, \quad u \in T, \quad s \neq t\right\} .
$$

We now put $\Sigma=\left\{(s u)(t u)^{-1}=s t^{-1} \mid s, t \in S, u \in T, s \neq t\right\}$. Then notice that $\Sigma$ is finite and that

$$
L_{2}=\mathrm{gp}_{B_{2}}\left\{\left[a_{s}, a_{t}^{\prime}\right] \mid a, a^{\prime} \in A, \quad s, t \in T \text { and } s t^{-1} \in \Sigma\right\} .
$$

Since $T$ is infinite and $\Sigma$ is finite, there exists $t_{0} \in T$ such that $t_{0} \notin \Sigma, t_{0}^{-1} \notin \Sigma$. By the very definition of $\Sigma, 1 \notin \Sigma$. We now put $\Delta=\left\{1, t_{0}\right\}$. Observe that

$$
\Delta \Delta^{-1} \cap \Sigma=\emptyset \text {. }
$$

We define the homomorphism

$$
\delta: B_{2}=\prod_{t \in T}^{(2)} A_{t} \longrightarrow \prod_{\delta \in \Delta}^{(2)} A_{\delta}
$$


by defining $\delta$ to be the identity homomorphism on $A_{1}$ and $A_{t_{0}}$ and the homomorphism which takes all of the other $A_{t}$ to the identity element. If $s t^{-1} \in \Sigma$, then either $s \notin \Delta$ or else $t \notin \Delta$. It follows that

$$
\left\{\left[a_{s}, a_{t}^{\prime}\right] \mid a, a^{\prime} \in A, \quad s, t \in T, \quad s t^{-1} \in \Sigma\right\}
$$

is in the kernel of $\delta$ and consequently so too is $L_{2}$. Hence $\delta$ induces a homomorphism of $B_{2} / L_{2}$ onto $\prod_{\delta \in \Delta}^{(2)} A_{\delta}$. Now $B_{2} / L_{2}$ is isomorphic to $B_{1}$, the restricted direct product of the $A_{t}, t \in T$, so if $a \in A$ is non-trivial, then $\left[a_{1}, a_{t_{0}}\right]=1$ in $B_{2} / L_{2}$ but, by Lemma I(3), $\left[a_{1}, a_{t_{0}}\right] \neq 1$ in $\prod_{\delta \in \Delta}^{(2)} A_{\delta}$. This contradiction completes the proof of Lemma 2.

5.4. The last step in the proof We have proved that if $A \neq 1$ and $W=A \imath T$ is of type $F P_{2}$, then $T$ is finite. Now a subgroup of finite index in a group of type $F P_{2}$ is again of type $F P_{2}$ (see Proposition 2.5 on page 20 of [4]). So $B_{2}$ is of type $F P_{2}$. Since $B_{2}$ is the direct product of finitely many copies of $A$, it follows that $A$ is of type $F P_{2}$, as required. This completes the proof of the theorem.

\section{References}

[1] G. Baumslag, 'A remark on generalized free products', Proc. American Math. Soc. 13 (1962), 53-54.

[2] — 'Wreath products and finitely presented groups', Math. Z. 75 (1960/1), 22-28.

[3] M. Bestvina and N. Brady, 'Morse theory and finiteness properties of groups', Invent. Math. 129 (1997), 445-470.

[4] R. Bieri, 'Homological group theory', Queen Mary College Mathematics Notes (1976).

[5] R. Bieri and R. Strebel, 'Valuations and finitely presented metabelian groups', Proc. London Math. Soc. (3) 41 (1980), 439-464.

[6] O. N. Golovin, 'Metabelian products of groups', Mat. Sbornik 27 (1950), 427-454. (English Translation: AMS Translations 2 (1956)), 117-132.

[7] K. W. Gruenberg, 'On the residual properties of infinite soluble groups', Proc. London Math. Soc. (3) 7 (1957), 29-62.

[8] F. W. Levi, 'The commutator group of a free product', J. Indian Math. Soc. 4 (1940), 136-144.

[9] A. L. Śmelkin, 'Wreath products and varieties of groups', Izvestiya Akad. Nauk. SSSR Ser. Mat. 29 (1965), 149-170 (in Russian).

Department of Mathematics

Mathematical Institute

City College of New York 24-29 St. Giles

Convent Avenue at 138th Street Oxford OX1 3LB

New York, NY 10031

U. K.

USA

e-mail: bridson@maths.ox.ac.uk

e-mail: gilbert@groups.sci.ccny.cuny.edu 


\section{Mathematics Department}

Queen Mary and Westfield College

Mile End Road

London E1

U.K.

e-mail: K.W.Gruenberg@qmw.ac.uk 